\title{
Ultraschall-Reflexionsmessungen zur Bestimmung der Füllstoffverteilung in Kunststoff-Compounds
}

\author{
Sebastian Wöckel ${ }^{1}$, Hendrik Arndt ${ }^{1}$, Ulrike Steinmann ${ }^{1}$, Jörg Auge ${ }^{1}$, Kilian Dietl², Giovanni Schober ${ }^{2}$, \\ Christoph Kugler' ${ }^{2}$, Thomas Hochrein ${ }^{2}$ \\ ${ }^{1}$ Institut für Automation und Kommunikation (ifak) e.V. Magdeburg, \\ Geschäftsfeld Messtechnik und Leistungselektronik, Werner-Heisenberg-Str. 1, 39106 Magdeburg \\ E-Mail: sebastian.woeckel@ifak.eu, Tel.: +49 3919901 430, Fax: +49 3919901590 \\ ${ }^{2}$ SKZ - Das Kunststoff-Zentrum \\ Prozessmesstechnik, Friedrich-Bergius-Ring 22, 97076 Würzburg
}

\section{Zusammenfassung}

Der Beitrag stellt die Kombination der Ultraschall-Reflexions-Methode mit einer statistischen Signalauswertung zur Charakterisierung der Dispergiergüte von Füllstoffen in Kunststoffschmelzen vor. Das Verfahren basiert auf der Erfassung der Streuung an bewegten Partikeln bei hohen Konzentrationen oder Medien hoher Grunddämpfung. Anhand der linearen Approximation der Standardabweichung der zeitabhängigen Amplitude der Reflexionssignale lassen sich zwei Kennwerte ( $\sigma_{\max }$ - Streuleistungsäquivalent und $\Delta \sigma$ - Dämpfungsäquivalent) ableiten. Diese korrelieren mit der Partikel-Streudämpfung und liefern ähnliche Informationen wie die Extinktionsdämpfung konventioneller Transmissionsverfahren ohne deren Limitierungen hinsichtlich des Messspalts.

Keywords: Partikelcharakterisierung, Polymerschmelze, Ultraschall, Streuverfahren

\section{Motivation}

In der Kunststoffindustrie sind Messverfahren gefordert, die es erlauben, kontinuierlich die Produktqualität zu überwachen [1]. Die meisten Kunststoffe bestehen aus einem Basispolymer und zusätzlich eingebrachten Füllstoffen bzw. Additiven, welche gezielt z. B. die mechanischen Eigenschaften, Leitfähigkeit, Entflammbarkeit oder Farbe des Materials beeinflussen. Diese werden als Compounds bezeichnet und bei den nachfolgenden formgebenden Prozessen zum Halbzeug- oder Endprodukt verarbeitet. Bei der Kunststoffaufbereitung wird das Basispolymer aufgeschmolzen und in einem Extruder mit den Feststoffpartikeln der Additive und Füllstoffe vermischt (Abb. 1). Typische Kunststoffcompounds enthalten Additive und Füllstoffe mit Partikelgrößen bis zu einigen $100 \mu \mathrm{m}$. Die Partikelverteilung dieser Füllstoffe in der Polymerschmelze stellt eines der zentralen Qualitätskriterien bei der Kunststoffaufbereitung dar.

Die bisher in der Praxis üblicherweise eingesetzten Analysemethoden sind arbeits- und zeitaufwändig und ermöglichen keine lückenlose $100 \%$-Prozesskontrolle [1, 2]. Es erfolgt entweder eine dem Produktionsprozess nachgeschaltete oder prozessparallele Analyse am Extruder. Die bevorzugte Methode ist hierbei anwendungsspezifisch.

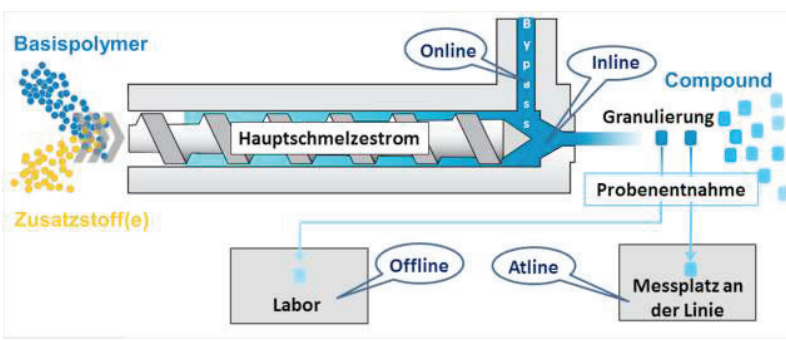

Abb. 1: Schema eines Extruders: Das Polymer (blau) wird durch Scherung in der Förderschnecke aufgeschmolzen und mit Füllstoffen und Additiven zum gezielten Verändern der gewünschten Materialeigenschaften vermischt.

Bei den Labormessungen zur Bestimmung der Materialkennwerte werden vorrangig optische oder Elektronenraster-Mikroskopie eingesetzt, die jedoch bei hohem Probenpräparationsaufwand nur einen ausgewählten Bereich des Polymers abbilden.

Alternativ kann über die Messung der Schlagoder Zugfestigkeit auf die Verteilung der Feststoffe im Compound (Dispergiergüte) geschlossen werden [1-3]. Neben der aufwändigen Probenvorbereitung führen hier Querempfindlichkeiten der mechanischen Eigenschaften bzgl. anderer Einflussgrößen zu einer ungenügenden Reproduzierbarkeit.

Die bislang einzige standardisierte Methode zur Bestimmung der Dispergiergüte stellt der Druckfiltertest nach DIN EN 13900-5 dar [4]. 
Diese Methode ist hauptsächlich für Pigmente $(<1 \mu \mathrm{m})$ und nicht für grobkörnige $(10 \ldots 100$ $\mu \mathrm{m})$ Füllstoffe geeignet. Zudem liefert sie keine Informationen zur Menge und Größe von Agglomeraten. Von dem Druckfiltertest existiert auch eine Online-Variante [1], die über einen Bypass am Extruder realisiert wird und zeitgleich zum Prozess stattfindet. $\mathrm{Zu}$ weiteren Prozessmessmethoden [1, 2] zählt u. a. die optische Kleinwinkellichtstreuung. Diese in anderen Industriebereichen etablierte Methode kann zur Partikelcharakterisierung von transluzenten Schmelzen mit zwei polymeren Phasen [3, 5] eingesetzt werden.

Eine weitere Inline-Methode stellt die Überwachung mit optischen CCD Zeilenkameras direkt am extrudierten Endprodukt dar. Hiermit lassen sich Fremdstoffe, Agglomerate und Inhomogenitäten detektieren, wobei die Transparenz des Polymers eine wesentliche Voraussetzung ist und somit vorrangig bei der Folienextrusion angewendet wird.

Alternative Inline-Ultraschall-Verfahren liefern anhand der in Transmission durch die Partikelstreuung erzeugten Extinktion eine Aussage über das Materialsystem [3, 6, 7] (Abb. 2). Der gemessene effektive Dämpfungsfaktor ist im Wesentlichen das Resultat von vier Dämpfungsmechanismen: der thermischen Partikelpulsation, der visko-inertialen Oszillation, der Absorption und der Streuung an den Partikeln. Die Herausforderung besteht darin, mit adäquaten Modellen [8] den Streuanteil, der mit der Größe, Anzahl und Verteilung der Partikel korreliert, zu trennen. Zur Verbesserung des gewünschten Streueffekts werden typischerweise Schallwellen mit Wellenlängen kleiner als die Partikelgröße verwendet (Abb. 3). Je größer das Partikel im Vergleich zur Wellenlänge wird, desto höher ist die erfasste Streudämpfung. Versuchsreihen zur Extinktionsdämpfung in Polymerschmelzen [9] in Abhängigkeit der Verteilung von Partikeln zweier Größenfraktionen $(1,4 \mu \mathrm{m}$ und $37 \mu \mathrm{m})$ zeigen, dass bereits auf einfache Weise mit Hilfe einer linearen Approximation das Vorhandensein bzw. der Anteil der "größeren" Partikelfraktion als Modellsystem für Agglomerate in der Schmelze überwacht werden kann (Abb. 4).

Im Gegensatz zu optischen Methoden [10] können diese Verfahren auch an hoch konzentrierten und opaken Schmelzen angewendet werden und sind für einen weiten Partikelgrößenbereich geeignet. Aufgrund der erheblichen Schalldämpfung der Polymermatrix in Kombination mit hoch konzentrierten Partikelsystemen ist es jedoch auch hier erforderlich, die Extinktionsverfahren auf BypassAnwendungen oder kleinere Laborextruder zu beschränken, was beim Anwender nur zu geringer Akzeptanz führt.

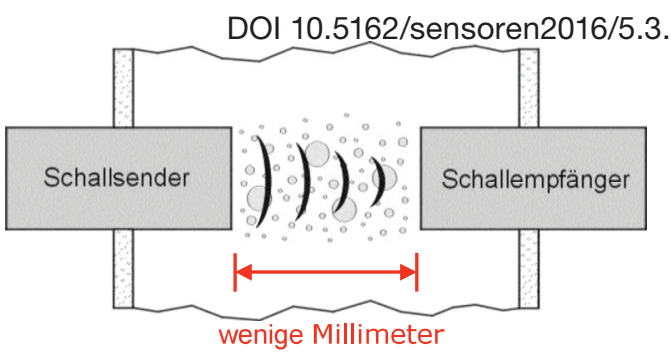

Abb. 2: Schematischer Aufbau einer USTransmissionsanordnung: Sender und Empfänger liegen sich dabei direkt gegenüber.

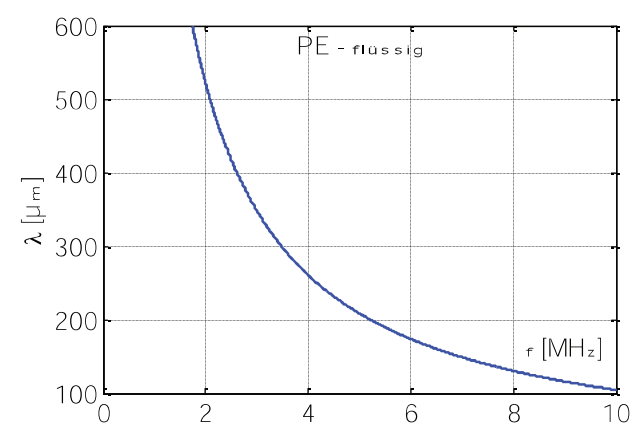

Abb. 3: Wellenlänge $\lambda$ in Polyethylen (PE flüssig) in Abhängigkeit von der Anregungsfrequenz $f$.

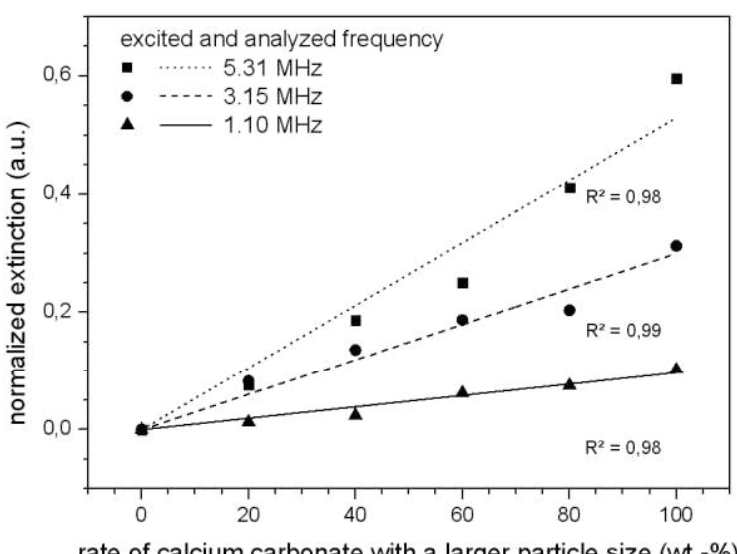

rate of calcium carbonate with a larger particle size (wt.-\%)

Abb. 4: Normierter Extinktionskoeffizient von Polypropylen in Abhängigkeit des gravimetrischen Anteils von Kreidepartikeln mit $37 \mu \mathrm{m}$ Durchmesser anstelle von Partikeln mit 1,4 $\mu m$ Durchmesser für drei ausgewählte Frequenzen bei konstantem Füllstoffanteil von $5 \%$ (vgl. Tab. 1) [9].

Tab. 1: Materialparameter

\begin{tabular}{|c|c|c|}
\hline Material & $\rho\left[\mathrm{kg} / \mathrm{m}^{3}\right]$ & $C_{\text {Longitudinal }}[\mathrm{m} / \mathrm{s}]$ \\
\hline PE (flüssig) & 920 & 1045 \\
\hline Hydrocarb. 95T & 2700 & 7400 \\
\hline $\begin{array}{c}\text { Spheriglass } \\
\text { 3000CP }\end{array}$ & 1590 & 6790 \\
\hline
\end{tabular}


Zur Lösung der genannten Probleme wird ein methodisch neuer Ansatz der statistischen Verarbeitung von Ultraschallreflexionsmessergebnissen [11] vorgestellt. Dabei wird anstelle der Transmission die an Füllstoffpartikeln zurückgestreute akustische Welle kontinuierlich und direkt im Kunststoffverarbeitungsprozess erfasst (Abb. 5). Gegenüber bekannten Transmissionsverfahren ermöglicht die Messung in Rückstreuung die Analyse höher konzentrierter Stoffsysteme. Die statistische Betrachtung der frequenzabhängigen Streuamplitude und deren Verringerung in Abhängigkeit der Zeit liefern Informationen zur Partikelkonzentration und -größenverteilung der Füllstoffe. Hierdurch bietet sich eine verfahrenstechnische Alternative zur aufwändigen Offline-Analytik.

Im Folgenden wird die Methode der statistischen Verarbeitung von Ultraschallstreusignalen an bewegten Kunststoffschmelzen vorgestellt und mit Inline-Messungen am Extruder an Polyolefinschmelzen aus Polypropylen (PP) und Polyethylen (PE) und Füllstoffen (Glaskugeln, Kreide) mit Partikelgrößen von $d=1 \ldots 100 \mu \mathrm{m}$ mit einem Feststoffgehalt bis $20 \mathrm{Ma} \%$ verifiziert.

\section{Rückstreuung und Standardabweichung}

Zur Charakterisierung von Dispersionen und Suspensionen existieren bereits Messverfahren bzw. prozessfähige Messgeräte, die auf der Analyse der Schallstreuung an einzelnen Partikeln beruhen [UPM Ultrasonic Particle Monitor, Netherlands Organization for Applied Scientific Research TNO], [Model AS3 Acoustic Sensor, Galvanic Applied Sciences Inc.]. Den gemessenen Streusignalen werden dabei Einzelechos, d. h. von einzelnen Partikeln reflektierte Schallwellen zugeschrieben. Diese Messgeräte sind für den Einsatz in sehr gering konzentrierten Partikelsystemen konzipiert, da sie einzelne und voneinander unabhängige Streuereignisse auswerten und meist zur Einzelpartikeldetektion genutzt werden.

Ähnlich der klassischen Reflexionsanordnung stehen bei der neuen Methode die Sende/Empfangswandler über eine Vorlaufstrecke im Kontakt mit der Dispersion. Die aktive Oberfläche der Wandler ist durch die Vorlaufstrecke aus temperaturbeständigem Kunststoff sowohl thermisch als auch abrasiv geschützt. Zum Einsatz kommen Wandler mit Mittenfrequenzen von $2 \ldots 5 \mathrm{MHz}$. Die Anregung erfolgt konventionell als Impuls (US-Pulser/Receiver UT340, Utex Scientific Instruments, Inc.) oder kodiertes Signal.

Das nachfolgend beschriebene Verfahren setzt voraus, dass sich die Verteilung der Partikel (mit statistisch zufälliger Bewegung) innerhalb des Schallfeldes und damit die Streucharakteristik zwischen zwei aufgenommenen Einzel-
DOI 10.5162/sensoren2016/5.3.2 signalen ändern. Dies ist i. d. R. beim Compoundierprozess durch den Massestrom gegeben. Letzteres bestimmt $u$. a. die maximal zulässige Messgeschwindigkeit.

Die Schallwellen $(f=2 \mathrm{MHz})$ haben in der flüssigen Polymerschmelze (z. B. PE), wie auch annähernd in der Dispersion, eine Wellenlänge $\lambda$ von etwa $250 \ldots 500 \mu \mathrm{m}$. Die Partikelgröße der Füllstoffe liegt mit $d=1 \ldots 100 \mu \mathrm{m}$ eine Größenordnung unterhalb der Wellenlänge. Entsprechend schwach ausgeprägt ist die Schallstreuung durch die Partikel [12]. Die Untersuchungen zeigen, dass selbst bei schwacher Streuung die messtechnische Erfassung und Auswertung von US-Reflexionsbzw. Rückstreusignalen möglich ist.
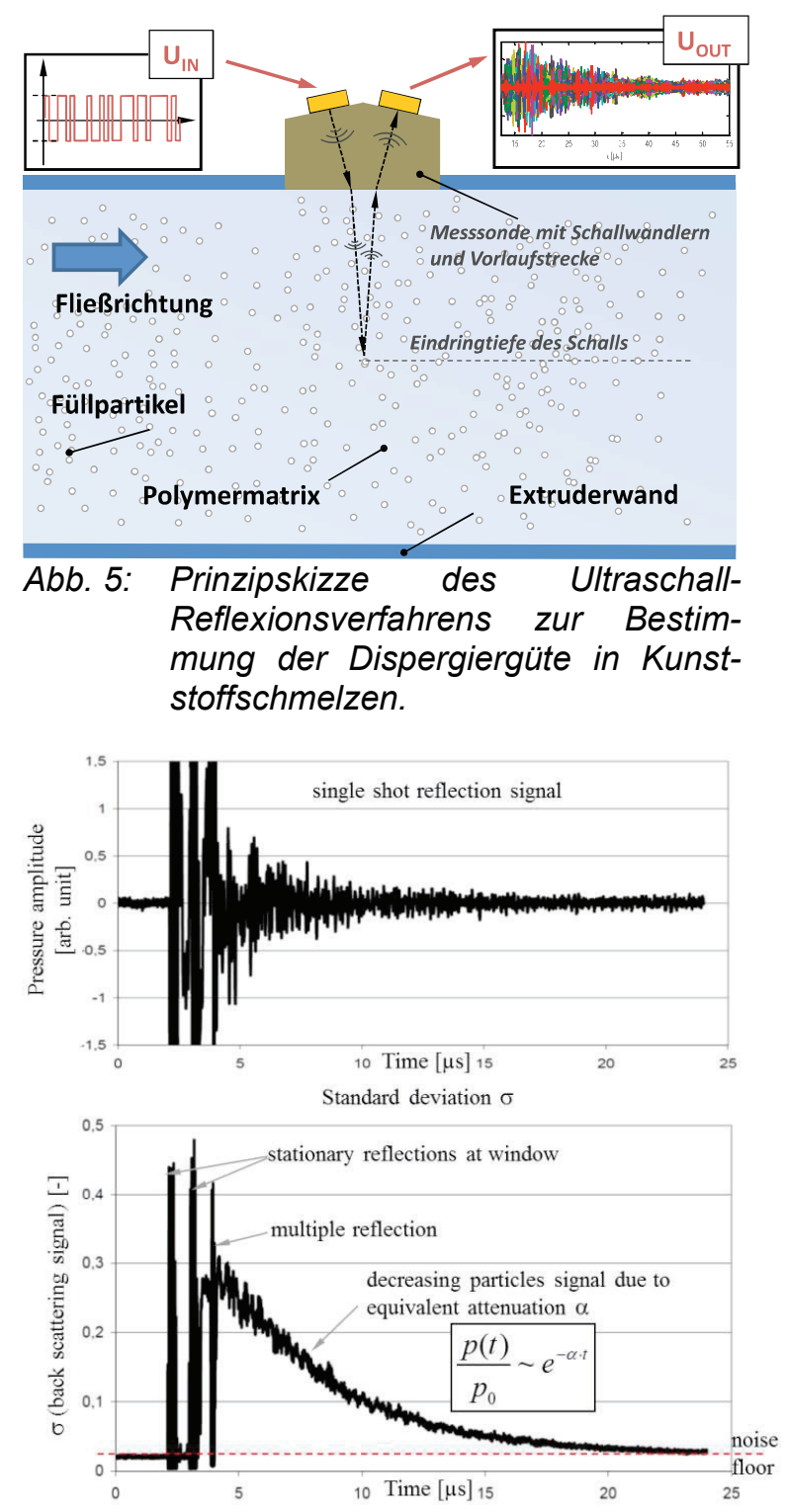

Abb. 6: US-Einzel-Rückstreusignal (oben) und laufzeitabhängige Standardabweichung $\sigma$ (unten) aus $N=200$ Einzelmessungen für eine Dispersion in Wasser mit $\mathrm{SiO}_{2}$ Partikeln (Partikelgröße $d<20 \mu \mathrm{m} ; \rho=2.35 \mathrm{~g} / \mathrm{cm}^{3}$; Konzentration 30 Ma\%). 
Die direkte Ultraschallrückstreumessung liefert nach Anregung des Schallwandlers ein Echosignal (Amplitude $p$ in Abhängigkeit von der Laufzeit $t$ ), das neben stationären Reflexionen ebenfalls die Rückstreusignale der Partikel enthält. Ein solcher Signalverlauf ist am Beispiel einer Dispersion aus Wasser mit $\mathrm{SiO}_{2}-$ Partikeln (Dichte: $2,35 \mathrm{~g} / \mathrm{cm}^{3}$, mittlere Partikelgröße: $3 \mu \mathrm{m}$ ) in Abb. 6, oben gezeigt.

Aus dem Echo- bzw. Rückstreusignal wird deutlich, dass die durch Partikel erzeugten Signalkomponenten aufgrund ihrer geringen Amplitude nur unzureichend von Signalanteilen infolge der (Mehrfach-) Reflexion und abgespaltenen Transversalmoden zu unterscheiden sind. Daher wurde anstelle der absoluten Auswertung der Echoamplitude deren Standardabweichung in Abhängigkeit von der Laufzeit herangezogen. Nach Erfassung von $\mathrm{N}$ Einzelmessungen (vollständiges Echosignal infolge eines ausgesendeten Impulses) wird zu jeder Laufzeit $t$ die Standardabweichung $\sigma$ der jeweils N Amplitudenwerte bestimmt. Statische Signalanteile würden hier keinen Anteil zur Standardabweichung liefern. Demgegenüber erzeugen die zeitlich veränderlichen Partikelsignale infolge der Bewegung der Partikel durch das Messvolumen eine deutliche Standardabweichung. Diese zeitabhängige Standardabweichung korreliert mit der Wahrscheinlichkeit der Messbarkeit der Streuung an einem Einzelpartikel zu einem bestimmten Zeitpunkt. Folglich repräsentiert eine hinreichende Anzahl von $\mathrm{N}$ Einzelmessungen die integrale homogene Verteilung des bewegten Partikelsystems. Mittels der statistischen Verarbeitung von mehreren Messsignalen lassen sich zudem das effektive Signal-zu-Rausch-Verhältnis und damit die Eindringtiefe verbessern.

\section{Auswertung und Verifizierung}

Beim Durchlaufen der Dispersion werden die emittierte und die an den Partikeln gestreuten Schallwellen in Abhängigkeit von der zurückgelegten Wegstrecke gedämpft. Die vom Schallwandler detektierbare Echosignalamplitude verringert sich demnach mit zunehmender Laufzeit - gleiches gilt für die Standardabweichung. Die Schallamplitude fällt dabei exponentiell mit der durchschallten Wegstrecke bzw. der Laufzeit ab und wird $u$. a. von der Partikelgröße und -konzentration bestimmt $[12,13]$ (vgl. Abb. 6 unten).

Zur Verifikation wurden Inline-Messungen am Laborextruder mit Polyolefinschmelzen (PP, $\mathrm{PE}$ ) und Füllstoffen (Glaskugeln, Kreide) mit Partikelgrößen von $d=1 \ldots 100 \mu \mathrm{m}$ mit einem Feststoffgehalt bis $20 \mathrm{Ma} \%$ durchgeführt. Abb. 7 und 8 zeigen den Extruder und die verwendeten Ultraschallsonden mit einer Mittenfrequenz von $2 \mathrm{MHz}$.

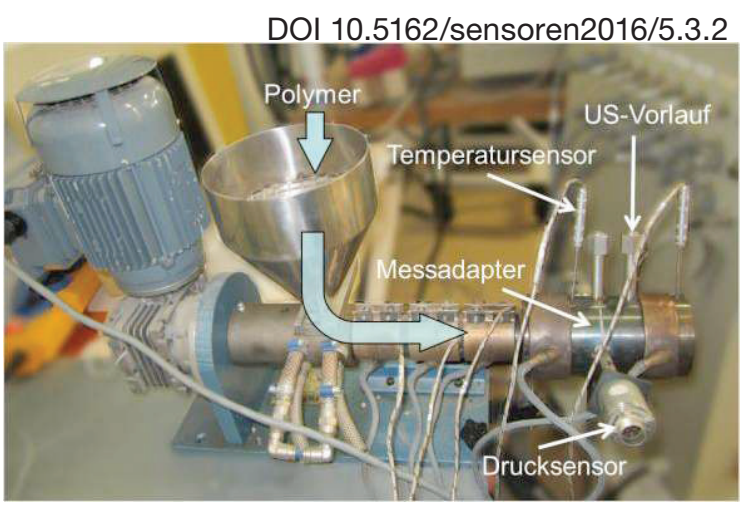

Abb. 7: Laborextruder und Messadapter mit Ultraschall-, Temperatur- und Drucksonden für Hochtemperaturanwendungen $\left(T<300^{\circ} \mathrm{C}\right)$.

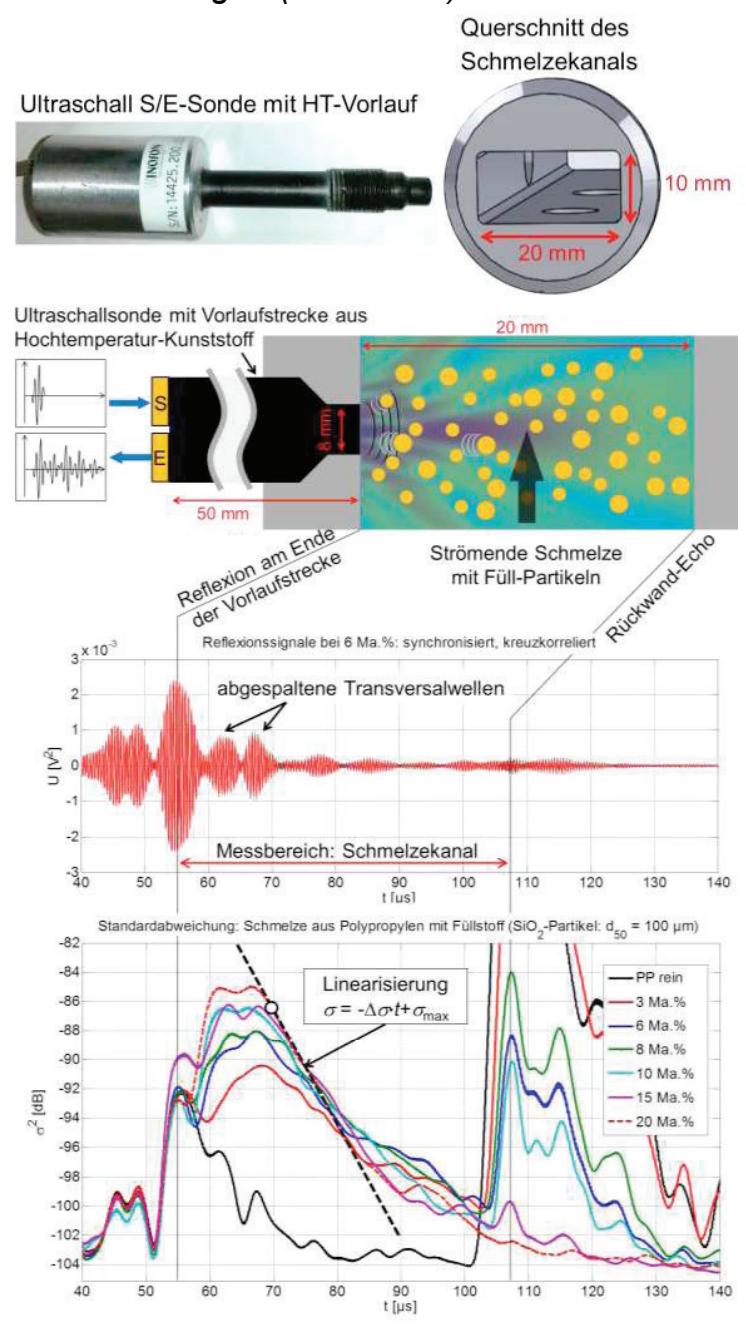

Abb. 8: US-Sonde und Schmelzekanal inklusive Dispersion (oben); Ultraschallreflexionssignale (unten) mit $N=500$ ( $f=2 \mathrm{MHz}$, Bandbreite $B=1 \mathrm{MHz}$ ) einer strömenden PolypropylenSchmelze (PP) $\left(T=286^{\circ} \mathrm{C}\right)$ mit $6 \mathrm{Ma} \% \mathrm{SiO}_{2}$-Partikeln (PartikelgröBe: $d 50=100 \mu \mathrm{m})$ und laufzeitabhängige Standardabweichung $\sigma$ (in [dB]) aus $N=500$ Einzelmessungen bei Variation der Partikelkonzentration von 3 bis 20 Ma.\% (Masseprozent). 
Bei logarithmischer Skalierung der Standardabweichung kann der in Abb. 6 und 8 gezeigte Verlauf abschnittsweise durch eine lineare Funktion approximiert werden:

$$
\sigma(t)[d B]=-\Delta \sigma \cdot t[\mu s]+\sigma_{\max }
$$

Die Standardabweichung $\sigma$ liefert damit einen einfachen Kennwert, dessen Amplitude (bzw. Maximum $\sigma_{\max } u$. a. von der Streuamplitude (d. h. Partikelgröße) und der Abfall der Kurve $\Delta \sigma$ von der Anzahl der Mehrfachstreuungen (d.h. Konzentration) abhängen. Mit Hilfe dieser zwei Kennwerte ist es bereits möglich, inline die Konzentration von Füllstoffpartikeln zu messen und im Bereich bis $10 \mathrm{Ma} . \%$ eineindeutig zuzuordnen (Abb. 9). Die Trennung für höhere Konzentrationen oder die Bewertung eines Gemischs aus verschiedenen Partikelgrößen (z. B. als Folge von Agglomerationen) erfordert die - hier nicht gezeigte - spektrale Verarbeitung der Daten in einem erweiterten Frequenzbereich [13].
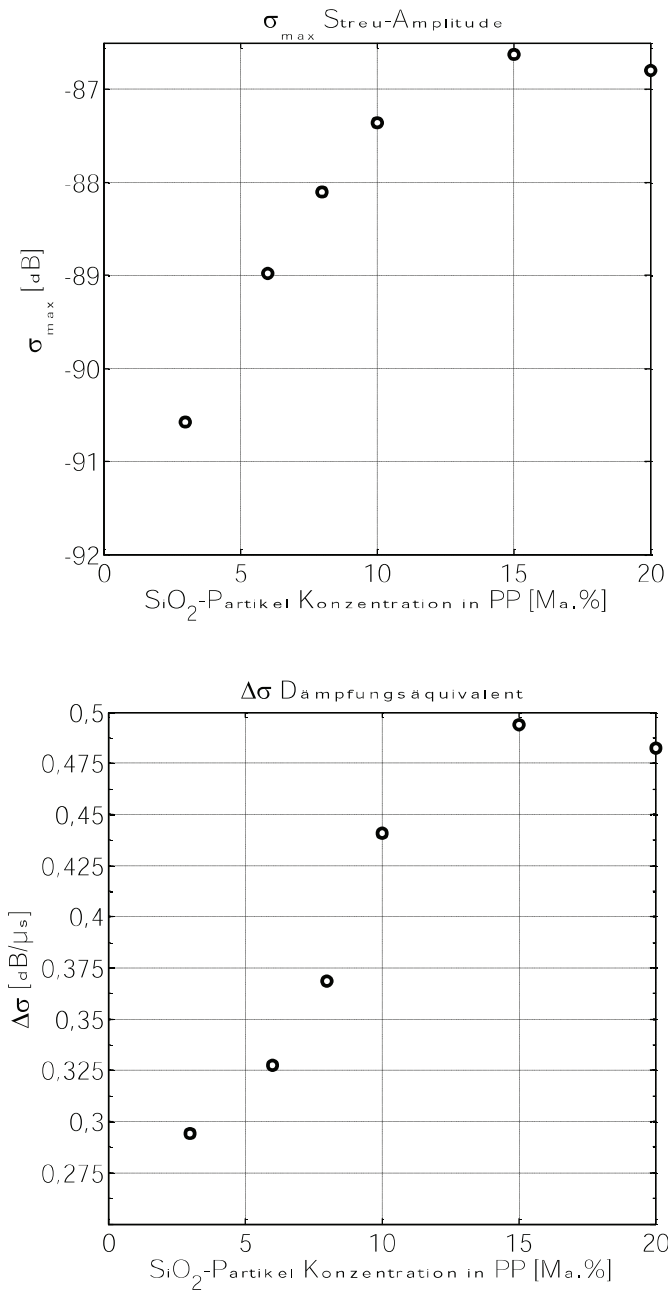

Abb. 9: Kennwerte $\left(\sigma_{\max }, \Delta \sigma\right)$ der Schmelze im Extruderkanal in Abhängigkeit der Partikelkonzentration; Materialdaten: $P P$ (flüssig): $\rho=920 \mathrm{~kg} / \mathrm{m}^{3}, \quad c_{L}=$ $1045 \mathrm{~m} / \mathrm{s}$, Spheriglass CP3000: $\rho=$ $1590 \mathrm{~kg} / \mathrm{m}^{3}, c_{L}=6790 \mathrm{~m} / \mathrm{s}$.

\section{Ausblick: Optimierung der Sonde}

Das erste Funktionsmuster der Sonde (Abb. 8) wurde gemäß der Vorgabe typischer Sensorzugänge am Extruder und in Anlehnung an bestehende Verfahren, die in Transmission arbeiten, ausgelegt. Für dieses Design treten jedoch neben der Reflexion am Ende der Vorlaufstrecke im Messbereich zusätzliche (statische) Störsignale in Folge der Abspaltung sekundärer Transversalwellen (T-Moden) an den Rändern im vorderen Bereich der Vorlaufstrecke auf. (Mit fortschreitender Welle bzw. Länge der Vorlaufstrecke gilt die Näherung planarer Wellenfronten mit geringer Kopplung zur Wand.) Bei Berechnung der Standardabweichung sollten diese statischen Signale keinen Einfluss zeigen, jedoch treten bereits durch geringe Temperaturschwankungen im Extruder $\left(+/-1{ }^{\circ} \mathrm{C}\right)$ Laufzeitänderungen (Phasenrauschen) auf, die einen Beitrag zur Standardabweichung liefern. Das temperaturabhängige Phasenrauschen der genutzten LMode lässt sich hinreichend durch eine Korrelation und Synchronisation kompensieren. Für die abgespaltenen T-Moden gelingt dies nicht. Für die Linearisierung der Standardabweichung ist es erforderlich, dass im Messbereich nach der L-Moden-Reflexion am Ende der Vorlaufstrecke vorrangig variable Signale auftreten, die mit der Partikelstreuung korrelieren.

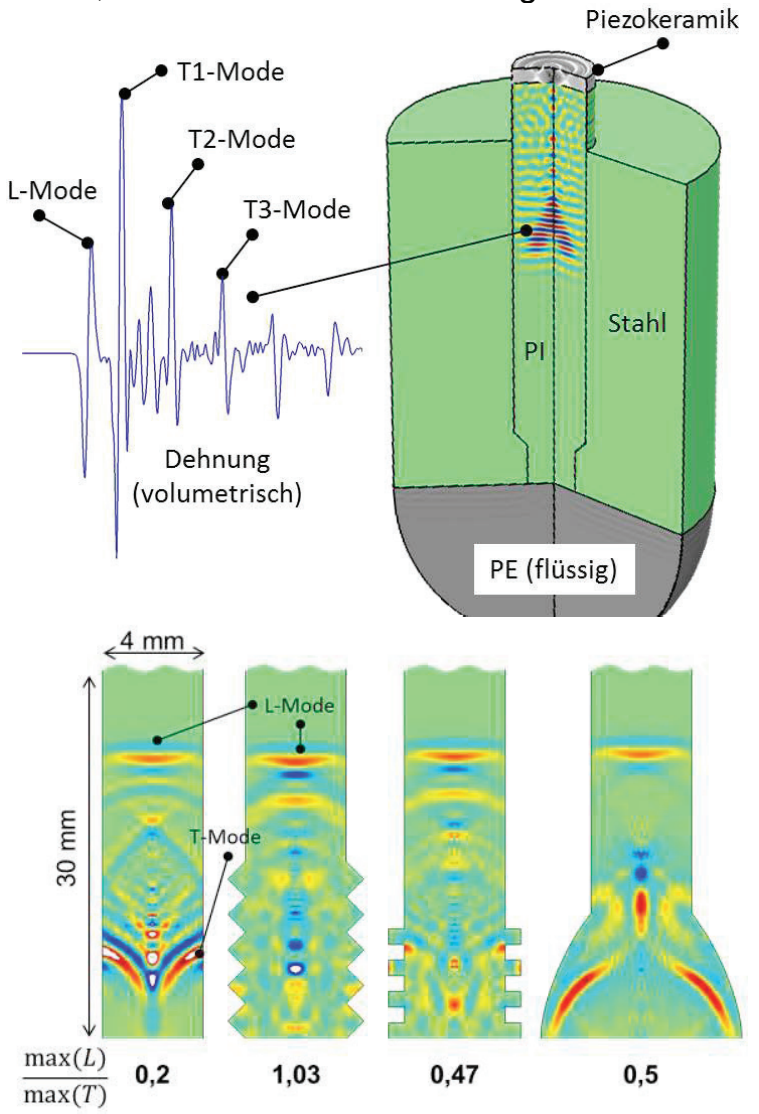

Abb. 10: 3D-Simulationsstudie der aktuellen Sonde (oben) und Intensitätsdarstellung im Querschnitt bei variabler Sondengeometrie (unten). 
Durch die geringere Laufzeit treten die $\mathrm{T}$ Moden im Messbereich auf und verhindern u. a. eine abschnittsweise Linearisierung (Abb. 8) zur ortsaufgelösten Partikelcharakterisierung - statt der bisher integralen Auswertung. Zur Verringerung der Störung ist es entsprechend notwendig, zeitlich kurze Messintervalle mit geringer Temperaturdrift zu wählen oder die Vorlaufstrecke hinsichtlich der T-ModenUnterdrückung neu zu optimieren (Abb. 10).

Abb. 10 zeigt eine 3D-Simulationsstudie des aktuellen Sondendesign (oben) inklusive der Illustration eines Ausschnitts der multimodalen Impulsantwort aus L-Moden und abgespaltenen T-Moden (ohne Dämpfung); und (unten) die Momentaufnahmen der Druckverteilung bei Anregung mit einem Gaussimpuls mit $1 \mathrm{MHz}-$ Mittenfrequenz für eine Auswahl angepasster Sondenformen zur diffusen Streuung der LT-Modenkonversion im vorderen Sondenbereich. Aus dieser einfachen Darstellung lässt sich anhand des Verhältnisses der Maximalamplituden der Moden $(\mathrm{L} / \mathrm{T})$ erkennen, dass eine Dreieckstruktur in Größenordnung der Wellenlänge geeignet ist, die T-Moden hinreichend zu unterdrücken.

\section{Zusammenfassung}

Der Beitrag stellt die Kombination der Ultraschall-Reflexions-Methode mit einer statistischen Signalauswertung zur Charakterisierung der Dispergiergüte von Füllstoffen in Kunststoffschmelzen vor. Das Verfahren basiert auf der Erfassung der Streuung an bewegten Partikeln bei hohen Konzentrationen oder Medien hoher Grunddämpfung. Anhand der linearen Approximation der Standardabweichung $\sigma$ der zeitabhängigen Amplitude der Reflexionssignale lassen sich zwei Kennwerte $\left(\sigma_{\max }\right.$ - Streuleistungsäquivalent und $\Delta \sigma$ - Dämpfungsäquivalent) ableiten. Diese repräsentieren den Einfluss der Partikel auf die Streudämpfung und liefern ähnliche Informationen wie die Extinktionsdämpfung konventioneller Transmissionsverfahren. Die statistische Verarbeitung mehrerer Signale liefert zudem ein homogenes Abbild des bewegten Partikelsystems.

Mit der Reduktion auf eine Messsonde entsteht ein einfacher Messaufbau, der nur den einseitigen Zugang zum Schmelzekanal am Extruder erfordert und die Probleme bekannter Transmissionsanordnungen umgeht.

Als Ergebnis der Arbeiten im Rahmen des geförderten Projektes liegen am ifak und SKZ zwei vollständig nutzbare Systeme vor, die die Ultraschallreflexions- und -transmissionsmessung an realen Polymerschmelzen erlauben. Das System ist mit einer Software ausgestattet, die neben der Inline-Messung am Extruder und Datenspeicherung gleichzeitig die direkte (online) Auswertung und Darstel-
DOI 10.5162/sensoren2016/5.3.2 lung der aktuellen Messergebnisse erlaubt. Messungen mit kodierten Sequenzen zur Erhöhung der Eindringtiefen für Industrieextruder mit größeren Querschnitten sind ebenfalls vorgesehen.

Im Rahmen von Messkampagnen an einem Laborextruder (ifak) und einem industrienahen Modellextruder (SKZ) liegen umfangreiche Messdaten für verschiedene Polymerschmelzen mit Ultraschall-, Temperatur- und Druckdaten vor. Mit diesen Daten werden erste Konzepte für die praxisrelevante Evaluierung und der Ableitung einer Dispergiergüte untersucht.

\section{Förderhinweis}

Das IGF-Vorhaben 18220 BG der Forschungsvereinigung Fördergemeinschaft für das SKZ e. V. wird über die AiF vom BMWi gefördert.

\section{Literaturnachweis}

[1] T. Hochrein, et.al., Prozessmesstechnik in der Kunststoffaufbereitung, Vogel-Verlag, Würzburg, 2011.

[2] I. Alig, et.al., Monitoring of polymer melt processing, MST 2010, 21, 1.

[3] B. Steinhoff, et.al., In-line Monitoring of the Dispersion of Nanofillers in Polymer Composites ..., Proc. PPS 21, 2005.

[4] DIN EN 13900-5:2005-07: Pigments and extenders - Methods of dispersion and assessment of dispersibility in plastics - Part 5: Determination by filter pressure value test, 2005

[5] S. Li, et.al, Light-scattering pho-tometer with optical microscope for the in-line study of polymer extrusion, Journal of Polymer Science Part B 1997, 35, 2282.

[6] G. Schober, et.al, Degree of dispersion monitoring by ultrasonic transmission technique and excitation of the transducer's harmonics Proceeding Polymer Processing Society 29, 2013.

[7] I. Alig, et.al., Frequency-dependence of ultrasonic velocity and attenuation in 2-phase composite systems with spherical scatterers, Journal of Applied Physics 1992, 72, 5565.

[8] R.E. Challis, et.al., Ultrasound techniques for characterizing colloidal dispersions, Rep. Prog. Phys. 68 (2005) 1541-1637.

[9] G. Schober, et.al., Degree of dispersion monitoring by ultrasonic transmission technique and excitation of the transducer's harmonics Proceeding Polymer Processing Society 29, 2013.

[10] S. Li, et.al, Light-scattering photometer with optical microscope for the in-line study of polymer extrusion, Journal of Polymer Science Part B 1997, 35, 2282.

[11] R. Weser, et.al, Ultrasonic backscattering method for in-situ characterisation of concentrated dispersions, Powder Technology, Volume 268, 2014, ISSN 0032-5910

[12] A.S. Dukhin, et.al., Ultrasound for Characterizing Colloids, Elsevier, 2002.

[13] R. Weser, Erfassung und Auswertung von Ultraschallechosignalen zur Charakterisierung konzentrierter Suspensionen. Dresden, Technische Universität, Dissertation, 2014 